\title{
Ecuación No-lineal Abstracta de Kirchhoff-Carrier en espacios de Banach
}

\author{
Raúl Izaguirre ${ }^{1}$ \\ rizaguirrem@unmsm.edu.pe \\ Ricardo Fuentes ${ }^{2} \quad$ Manuel Milla ${ }^{3}$
}

\begin{abstract}
Resumen
Sean $(V, a(u, v)),(H,(u, v))$ espacios de Hilbert con inmersión $V \subset H$ densa y compacta. Consideramos el problema abstracto siguiente

$$
(*) \quad\left\{\begin{array}{l}
B u^{\prime \prime}+M\left(|u|_{0},\left|u^{\prime}\right|_{1}^{2}\right) A u=f \quad \text { en } \quad V^{\prime} \\
u(0)=u_{0} ; \quad u^{\prime}(0)=u_{1}
\end{array}\right.
$$

donde $B: H \rightarrow H$ es un operador lineal simétrico y positivo $;\left(W_{0},|u|_{0}\right),\left(W_{1},|u|_{1}\right)$ son espacios de Banach. $M$ es una función real a dos variables de clase $C^{1}$ y no negativa. En el trabajo se demuestra existencia local y unicidad de solución del problema $(*)$.
\end{abstract}

Palabras Clave: Kirchhoff-Carrier, Espacios de Banach

\section{Introducción}

Sean $(V, a(u, v)),(H,(u, v))$, espacios de Hilbert, $V \subset H$, la inmersión de $V$ en $H$ es densa y compacta. Sea $A$, el operador definido por la terna $\{V, H, a(u, v)\}$. Entonces, $A$ es un operador no-acotado, auto-adjunto y positivo de $H$, con espectro discreto. Asimismo para todo $\alpha \in \mathbb{R}$, el operador $A^{\alpha}$ esta bien definido. En este contexto $V=D\left(A^{1 / 2}\right)$.

El modelo abstracto

$$
\left\{\begin{array}{l}
u^{\prime \prime}+M\left(\left|A^{\alpha} u\right|^{2}\right) A^{\beta} u=f \\
u(0)=u_{0} \quad ; u^{\prime}(0)=u_{1}
\end{array}\right.
$$

considera como casos particulares los siguientes problemas:

\footnotetext{
${ }^{1}$ UNMSM, Facultad de Ciencias Matemáticas, Lima - Perú.

${ }^{2}$ Instituto de Matemática. UFF-Brasil.

${ }^{3}$ Instituto de Matemática. UFRJ - Brasil.
} 
Sea $\Omega$ un abierto de $\mathbb{R}^{n}$ con frontera regular $\Gamma$; Q el cilindro $\Omega$ x $] 0, \mathrm{~T}[0<\mathrm{T}<\infty$ con frontera lateral $\Sigma=\Gamma \times] 0, T]$. La ecuación diferencial parcial no-lineal

$$
\begin{cases}\rho(x) u_{t t}+\left(1+\int_{\Omega}|\nabla u|^{2} d x\right)(-\Delta u)=f(t) & \text { en } \mathrm{Q} \\ u=0 & \text { en } \Sigma \\ u(0)=u_{0} ; u_{t}(0)=u_{1} & \text { en } \Omega\end{cases}
$$

es un modelo generalizado de la ecuación de Kirchhoff estudiado en [8], planteada para estudiar las vibraciones de pequeña amplitud, de una cuerda fija en sus extremos y cuando la dependencia de la tensión no puede dejarse de lado en el modelo.

Asimismo el problema mixto

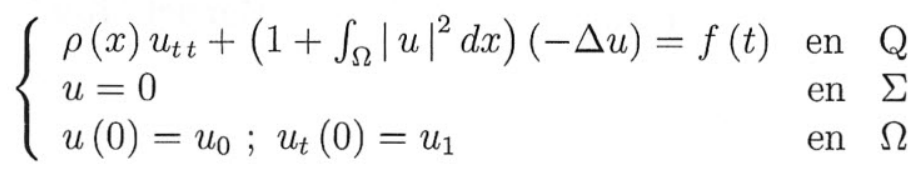

Es una generalización de un problema estudiado por Carrier en [2].

En [18] Pohozaev, trata el sistema

$$
\begin{cases}u^{\prime \prime}+(-1)^{m}\left(1+\int_{\Omega}\left|\nabla^{m} u\right|^{2} d x\right) \Delta^{m} u=f & \text { en } \\ \gamma_{0} u=\gamma_{1} u=\ldots \ldots \ldots=\gamma_{m-1}=0 & \text { en } \Sigma \\ u(0)=u_{0} ; u^{\prime}(0)=u_{1} & \text { en } \Omega\end{cases}
$$

Obsérvese que si $V=H_{0}^{1}(\Omega), H=L^{2}(\Omega), A=-\Delta, \alpha=1 / 2, \beta=1$, estamos en la Ecuación de Kirchhoff [10] ; si $\alpha=0, \beta=1$, es el modelo de Carrier [2] ; si $V=$ $H_{0}^{m}(\Omega), H=L^{2}(\Omega), A=-\Delta ; \alpha=m / 2, \beta=m$, es el modelo de Pohozaev. En los casos señalados $M(s)=1+s^{2}$.

Con relación a esta formulación, se plantea entre otros problemas, el estudio de la ecuación (1.1) para el caso en que la función no-lineal $M$ sea no-negativa (caso degenerado). La existencia y unicidad de soluciones locales para el caso degenerado de la ecuación de Kirchhoff $\alpha=1 / 2$ y $\beta=1$, son obtenidas por ejemplo en Ebihara -.Medeiros -Milla [5], asumiendo que $M \in C^{1}$ y que $\left|M^{\prime}(s) s\right| \leq a M(s)$, donde la constante $a$ es positiva. En [4] Crippa trata un caso bastante general suponiendo que la función $M \in C^{1}([0, \infty)), M(0)=0 \mathrm{y}$ $\forall \delta \succ 0, \quad \inf _{s \geq \delta} M(s) \succ 0$, demostrando que existe una única solución local débil al problema de Cauchy asociado a (1.1). En relación al modelo de Kirchoff- Carrier (1.3), se tiene las referencias [3] y [7], y donde la función no-lineal $\mathrm{M}$ es de clase $C^{1}$ y estrictamente positiva. En [3], se obtiene soluciones globales con datos analíticos "suficientemente pequeños". En [7], se obtiene solución local, pero en una clase mayor de datos iniciales. Para el caso degenerado se tiene la referencia [5] donde $\mathrm{M}$ es de clase $C^{1}$ y verifica cierta condición de crecimiento polinomial.

Una generalización del modelo (1.3) se da en la ecuación

$$
K u^{\prime \prime}(t)+M\left(|B u(t)|^{2}\right) A u(t)=f(t)
$$

donde $K$ y $B$ son operadores que conmutan con el operador $A$ y cumplen ciertos requisitos técnicos . 
Por ejemplo, para tratar el caso

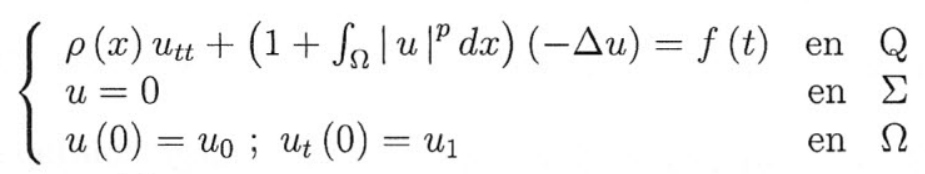

Se tiene la referencia [6], donde se obtiene solución local para el modelo abstracto

$$
\left\{\begin{array}{l}
B u^{\prime \prime}+M\left(|u|_{W}^{\beta}\right) A u=f \\
\text { en } V^{\prime} \\
u(0)=u_{0} \neq 0 ; u^{\prime}(0)=u_{1}
\end{array}\right.
$$

Donde $V$ es un espacio de Hilbert, con dual $V^{\prime}$ y $A, B: V \rightarrow V^{\prime}$ son operadores lineales simétricos con $\langle A v, v\rangle \geq 0,\langle B u, u\rangle>0, u \neq 0$. $W$ es un espacio de Banach con $V \subset W$ con inmersión contínua ; $\beta$ es un número real , $\beta \geq 1$ y $M(\xi)$, una función no negativa y regular en una vecindad de $\left|u_{0}\right|_{W}$.

Otra manera de tratar unificadamente los sistemas (1.1), (1.4) y (1.7), es considerar el modelo

$$
u^{\prime \prime}(t)+M\left(\left|A^{\alpha} u(t)\right|^{2},\left|A^{\beta} u(t)\right|^{2}\right) A^{\gamma} u(t)=f(t)
$$

donde la función no-lineal $M(s, r)$ es no- negativa y de clase $C^{1}$ en las dos variables. En la referencia [7] Izaguirre - García demuestran la existencia de solución para el caso $\alpha=1 / 2 ; \beta=0 ; \gamma=1$, y en la referencia [6] de Izaguirre-Fuentes-Milla, se demuestra la existencia de solución para un caso más general, que en otros trabajos del autor a ser publicados. Una extensión natural de los casos tratados es considerar la ecuación

$$
\left\{\begin{array}{l}
u^{\prime \prime}+M\left(\left|A^{\alpha} u\right|^{2},\left|A^{\beta} u^{\prime}\right|^{2}\right) A^{\gamma} u=f \\
\text { en } V^{\prime} \\
u(0)=u_{0} \\
u^{\prime}(0)=u_{1}
\end{array}\right.
$$

En la referencia [12] Long trata la ecuación de onda no lineal, con condiciones de frontera mixtas, según el siguiente modelo

$$
\begin{cases}u_{t t}+M\left(|u|^{2},|\nabla u|^{2}\right)(-\Delta u)=f(t) & \text { en } \mathrm{Q} \\ u=0 & \text { en } \Sigma \\ \frac{\partial u}{\partial \nu}+h u=0 & \text { en } \Sigma \\ u(0)=u_{0} ; u_{t}(0)=u_{1} & \text { en } \Omega\end{cases}
$$

donde $\Omega$ es la bola abierta unitaria en dimensión $\mathrm{N}=1,2$.

Una natural generalización de estos problemas es considerar el caso

$$
B u^{\prime \prime}+M\left(|u|_{0},\left|u^{\prime}\right|_{1}^{2}\right) A u=f, \text { en } V^{\prime} \quad u(0)=u_{0} \quad ; \quad u^{\prime}(0)=u_{1}
$$

Donde $|u|_{0},|u|_{1}$ son las normas en espacios de Banach convenientes. 


\section{Descomposición Espectral del Operador $T$}

Sean $(V, a(u, v)),(H,(u, v))$ espacios de Hilbert reales con inmersión $V \subset H$ densa y compacta.

Sea $b: H \times H \rightarrow \mathbb{R}$, una forma bilineal, simétrica (luego continua) y positiva, esto es :

$$
\begin{gathered}
b(u, v)=b(v, u) \quad u, v \in H \\
b(u, u)<0, \quad u \neq 0
\end{gathered}
$$

Entonces $(H, b(u, v))$ es un espacio pre- Hilbert . Sea $(\hat{H}, \hat{b}(u, v))$ su completación que es un espacio de Hilbert. Observemos que $b(u, v)=\hat{b}(i u, i v), \forall u, v \in H$, donde $i: H \rightarrow \hat{H}$ es el operador de inmersión que es lineal, isométrico de rango denso.

Entonces $V \subset \hat{H}$, con inmersión densa continua y compacta. Sea $S$ el operador lineal, autoadjunto determinado por la terna $\{V, \hat{H}, a(u, v)\}$. Se tiene que: $S: D(S) \subset V \rightarrow \hat{H}$ es biyectivo, con operador inverso $S^{-1}: \hat{H} \rightarrow D(S)$ compacto, $D(S)$ es denso en $V$ y en $\hat{H}$. Además se verifica la relación

$$
\hat{b}(S u, i v)=a(u, v) \quad, u \in D(S), v \in V .
$$

Observación 1. El espacio $\hat{H}$ es isomorfo al espacio dual fuerte $H^{\prime}$ de $(H, b(u, v)) \quad H$ y la inmersión $i: H \rightarrow H^{\prime}$ puede ser identificada con $B: H \rightarrow H^{\prime}$.

De acuerdo a la Teoria Espectral para operadores autoadjuntos y compactos, existe una sucesión ortonormal $\left\{w_{k}\right\}$ de autovectores de $S^{-1}$, para los cuales la correspondiente sucesión de autovalores $\left\{\mu_{k}\right\}$ converge a cero y los autovectores forman una base para $R g\left(S^{-1}\right)=D(A)$. Entonces tenemos que existe una base numerable $\left(w_{k}\right)_{k \geq 1}$ de $V$ y una sucesión creciente de números positivos $\left(\lambda_{k}\right)_{k \geq 1}$ tales que:

$$
\begin{gathered}
\lambda_{j} b\left(w_{j}, v\right)=a\left(w_{j}, v\right) \quad \forall v \in V ; j=1,2, \ldots \ldots \\
b\left(w_{j}, w_{i}\right)=\delta_{i j} ; a\left(w_{j}, w_{j}\right)=\lambda_{j} ; j=1,2, \ldots \ldots \\
S w_{j}=\lambda_{j} w_{j} ; j=1,2, \ldots
\end{gathered}
$$

Según la Teoría Espectral para $\alpha \in \mathbb{R}$, podemos definir la potencia $S^{\alpha}$, con dominio

$$
\begin{aligned}
& D\left(S^{\alpha}\right)=\left\{u ; \sum_{v=1}^{\infty} \lambda_{v}^{2 \alpha}\left|b\left(u, w_{v}\right)\right|^{2}<\infty\right\} \\
& S^{\alpha} u=\sum_{v=1}^{\infty} \lambda_{v}^{\alpha} b\left(u, w_{v}\right) w_{v} \quad \forall u \in D\left(S^{\alpha}\right) \\
& (u, v)_{\alpha}=\sum_{v=1}^{\infty} \lambda_{v}^{2 \alpha} b\left(u, w_{v}\right) b\left(v, w_{\nu}\right) \\
& |u|_{\alpha}^{2}=\left|S^{\alpha} u\right|^{2}=\sum_{v=1}^{\infty} \lambda_{v}^{2 \alpha}\left|b\left(u, w_{v}\right)\right|^{2}
\end{aligned}
$$

se tiene que $\left(D\left(S^{\alpha}\right),(u, v)_{\alpha}\right)$ es un espacio de Hilbert y sí $\alpha<\beta$ la inmersión de $D\left(S^{\beta}\right) \subset D\left(S^{\alpha}\right)$ es compacta $\forall \alpha, \beta \in \mathbb{R}$. 


\section{Problema Lineal}

Sea $T>0$. Consideremos el problema de hallar solución para el sistema

$$
\left\{\begin{array}{l}
B u^{\prime \prime}(t)+M(t) A u(t)=B f(t) \\
u(0)=u_{0} \quad ; u^{\prime}(0)=u_{1}
\end{array}\right.
$$

en $V^{\prime}$

Trabajando formalmente, sea $v \in V$. Entonces

$$
b\left(u^{\prime \prime}(t), v\right)+M(t) a(u(t), v)=b(f(t), v)
$$

Por (2.3)

$$
\hat{b}\left(i u^{\prime \prime}(t), i v\right)+M(t) \hat{b}(S u(t), i v)=\hat{b}(i f(t), i v)
$$

Entonces desde que $i v$ es denso en $\hat{H}$, el sistema es equivalente con

$$
\left\{u^{\prime \prime}(t)+M(t) S u(t)=f(t) \quad u(0)=u_{0} \quad ; u^{\prime}(0)=u_{1}\right.
$$

en $H^{\prime}$

\subsection{Problema (Solución Global)}

Sea $T>0 y$

$$
\begin{gathered}
\psi \in C^{1}([0, T]), \psi(t) \geq m_{0}>0 \quad \forall s \in[0, T] ; \\
\psi \in L^{\infty}(0, T) ; \quad|\psi|_{L^{\infty}(0, T)} \leq M_{1} .
\end{gathered}
$$

Teorema 1. Sea $\alpha \in \mathbb{R}$ y $\psi$ que verifica las condiciones (3.3) y (3.4).

$$
\begin{gathered}
u_{0} \in D\left(S^{\alpha+1}\right) \\
u_{1} \in D\left(S^{(2 \alpha+1) / 2}\right) \\
f \in L^{\infty}\left(0, T ; D\left(S^{(2 \alpha+1) / 2}\right)\right)
\end{gathered}
$$

Entonces existe una única solución $u$ del problema (3.1) tal que:

$$
\begin{gathered}
u \in L^{\infty}\left(0, T ; D\left(S^{\alpha+1}\right)\right) \\
u^{\prime} \in L^{\infty}\left(0, T ; D\left(S^{(2 \alpha+1) / 2}\right)\right) \\
u^{\prime \prime} \in L^{\infty}\left(0, T ; D\left(S^{\alpha}\right)\right) \\
u^{\prime \prime}+\psi(t) S u=f \quad \text { en } L^{\infty}\left(0, T ; D\left(S^{\alpha}\right)\right)
\end{gathered}
$$


La solución satisface el siguiente estimado de energía:

$$
E(t)=\left|S^{\alpha} u^{\prime}(t)\right|^{2}+\left|S^{2 \alpha+1 / 2} u(t)\right|^{2} \leq D_{0} \exp \left(D_{1} t\right)
$$

donde

$$
\begin{gathered}
C_{0}=\left|S^{(2 \alpha+1) / 2} u_{1}\right|^{2}+\psi(0)\left|S^{\alpha+1} u_{0}\right|^{2}+T|f|_{L^{\infty}\left(0, T ; D\left(S^{(2 \alpha+1) / 2}\right)\right)}^{2} \\
C_{1}=\min \left\{1, m_{0}\right\} ; \\
C_{2}=\operatorname{Max}\left\{1, M_{0}\right\} ; \\
D_{0}=\frac{C_{0}}{C_{1}} ; D_{1}=\frac{C_{2}}{C_{1}} .
\end{gathered}
$$

Demostración . Sea $V_{m}=\left[w_{1}, w_{2}, \ldots \ldots . ., w_{m}\right] \subset V$. Luego $V m$ es un subespacio de $V$ de dimensión finita m, e invariante bajo la acción del operador $S^{k}$.

Sea entonces

$$
u_{m}(t)=\sum_{i=1}^{m} g_{i m}(t) w_{i} \in \dot{V}_{m}
$$

Donde las funciones $g_{i m}$, son determinadas por la solución del siguiente sistema de ecuaciones diferenciales ordinarias lineales

$$
\left\{\begin{array}{l}
b\left(u_{m}^{\prime \prime}(t), w_{j}\right)+\psi(t) a\left(u_{m}(t), w_{j}\right)=a\left(f(t), w_{j}\right) \quad \forall j=1,2, \ldots, m \\
u_{m}(0)=u_{0 m} ; u_{m}^{\prime}(0)=u_{1 m}
\end{array}\right.
$$

Por la selección de la base especial, el sistema es equivalente con el sistema lineal de ecuaciones diferenciales ordinarias

$$
\left\{\begin{array}{l}
g_{j m}^{\prime \prime}(t)+\psi(t) \sum_{k=1}^{m} \lambda_{k} g_{k m}(t)=b\left(f(t), w_{j}\right)=f_{j}(t) \\
g_{j m}(0)=b\left(u_{0}, w_{j}\right)=a_{j} \\
g_{j m}^{\prime}(0)=b\left(u_{1}, w_{j}\right)=b_{j} \\
j=1,2 \ldots \ldots . m .
\end{array}\right.
$$

El sistema de ecuaciones diferenciales lineales (3.15), admite una única solución en un intervalo $\left[0, t_{m}\right\rangle$, de donde seguimos la existencia de las soluciones aproximadas $u_{m}$ para $m \geq 1$. Seguidamente debemos obtener estimados a priori para la sucesión $\left\{u_{m}\right\}\{u m\}$ de modo que podamos prolongarlas a un intervalo uniforme de existencia .

\subsection{Estimado a Priori 1}

Por linealidad la igualdad en (3.14) se verifica para todo $v \in V_{m}$. Entonces haciendo $v=S^{2 \alpha+2} u_{m}^{\prime}(t)$, obtenemos

$$
\left(S^{\alpha+1 / 2} u_{m}^{\prime \prime}(t), S^{\alpha+1 / 2} u_{m}^{\prime}(t)\right)+\psi(t)\left(S^{\alpha+1} u_{m}(t), S^{\alpha+1} u_{m}^{\prime}(t)\right)=\left(S^{\alpha+1 / 2} f(t), S^{\alpha+1 / 2} u_{m}^{\prime}(t)\right)
$$


Luego

$\frac{d}{d t}\left\{\left|S^{\alpha+1 / 2} u_{m}^{\prime}(t)\right|^{2}+\psi(t)\left|S^{\alpha+1} u_{m}(t)\right|\right\} \leq\left|S^{\alpha+1 / 2} f(t)\right|^{2}+\left|S^{\alpha+1 / 2} u_{m}^{\prime}(t)\right|^{2}+M_{1}\left|S^{\alpha+1} u_{m}(t)\right|^{2}(3$

Ahora integrando en esta desigualdad obtenemos

$$
\begin{aligned}
& \tau(t)=\left|S^{\alpha+1 / 2} u_{m}^{\prime}(t)\right|^{2}+m_{0}\left|S^{\alpha+1} u_{m}(t)\right|^{2} \\
& \leq\left|S^{\alpha+1 / 2} u_{m}^{\prime}(t)\right|^{2}+\psi(t)\left|S^{\alpha+1} u_{m}(t)\right|^{2} \\
& \leq \int_{0}^{t}\left|S^{\alpha+1 / 2} f(s)\right|^{2} d s+\int_{0}^{t}\left|S^{\alpha+1 / 2} u_{m}^{\prime}(s)\right|^{2} d s+\left|S^{\alpha+1 / 2} u_{m}^{\prime}(0)\right|^{2} \\
&+\psi(0)\left|S^{\alpha+1} u_{m}(0)\right|^{2}+M_{1} \int_{0}^{t}\left|S^{\alpha+1} u_{m}(s)\right|^{2} d s \\
& \leq\left|u_{1}\right|_{\alpha+1 / 2}^{2}+M_{0}\left|u_{0}\right|_{\alpha+1}^{2}+T|f|_{L^{\infty}\left(0, T ; D\left(S^{\alpha+1 / 2}\right)\right)}^{2}+\int_{0}^{t}\left|S^{\alpha+1 / 2} u_{m}^{\prime}(s)\right|^{2} d s \\
&+M_{1} \int_{0}^{t}\left|S^{\alpha+1} u_{m}(s)\right|^{2} d s \\
& \leq C_{0}+C_{2} \int_{0}^{t} \tau(s) d s
\end{aligned}
$$

Aplicando el lema de Gronwall obtenemos

$$
\left|S^{\alpha+1 / 2} u_{m}^{\prime}(t)\right|^{2}+\left|S^{\alpha+1} u_{m}(t)\right|^{2} \leq \frac{C_{0}}{C_{1}} e^{\dot{C}_{2} t / C_{1}}=D_{0} e^{D_{1} t}
$$

luego

$$
\begin{aligned}
& \left(u_{m}\right) \quad \text { es acotada en } L^{\infty}\left(0, T ; D\left(S^{\alpha+1}\right)\right) \\
& \left(u_{m}^{\prime}\right) \text { es acotada en } L^{\infty}\left(0, T ; D\left(S^{\alpha+1 / 2}\right)\right)
\end{aligned}
$$

\subsection{Estimado a Priori 2}

Multiplicando en la ecuación aproximada por $w_{j}$ y luego sumando, obtenemos

$$
\sum_{j=1}^{m}\left(u_{m}^{\prime \prime}(t), w_{j}\right) w_{j}+\psi(t) \sum_{j=1}^{m}\left(S u_{m}(t), w_{j}\right) w_{j}=\sum_{j=1}^{m}\left(f(t), w_{j}\right) w_{j}
$$

Entonces por definición de la proyección se obtiene

$$
\begin{array}{r}
u_{m}^{\prime \prime}(t)=-\psi(t) S u_{m}(t)+P_{m} f(t)= \\
=-\psi(t) S u_{m}(t)+f_{m}(t)
\end{array}
$$

Luego

$$
\left(u_{m}^{\prime \prime}\right) \quad \text { es acotada en } \quad L^{\infty}\left(0, T ; D\left(S^{\alpha}\right)\right)
$$

y

$$
\left|S^{\alpha} u_{m}^{\prime \prime}(t)\right| \leq M_{0}\left|S^{\alpha+1} u_{m}(t)\right|+d_{4}\left|S^{\alpha+1 / 2} f(t)\right|
$$




\section{Convergencia de las Soluciones Aproximadas}

Para tratar la convergencia de las soluciones aproximadas utilizamos el siguiente

Lema 2. Sean $X, Y, Z$ espacios de Banach tales que $X \subset Y \subseteq Z$, con inmersiones continuas y la inmersión $X \subset Y$ es compacta. Sea

$$
W=\left\{u \in L^{p}(0, T ; X) ; u^{\prime} \in L^{q}(0, T ; Z)\right\}
$$

donde $u^{\prime}$ denota la derivada generalizada de $u:[0, T] \rightarrow X$, sobre $(0, T)$. Entonces

$$
\begin{aligned}
& \text { Si } p=\infty, q>1 \text { entonces } W \subseteq C([0, T] ; Z) \text { es compacta. } \\
& \text { Si } 1 \leq p<\infty, q=1 \text { entonces } W \subseteq L^{p}(0, T ; Y) \text { es compacta. }
\end{aligned}
$$

Demostración . ( Ver J. Simon [20] ).

Entonces, por los estimados 1,2, tenemos que:

$$
\begin{gathered}
\left(u_{m}\right) \quad \text { es acotada en } L^{\infty}\left(0, T ; D\left(S^{\alpha+1}\right)\right) \\
\left(u_{m}^{\prime}\right) \quad \text { es acotada en } L^{\infty}\left(0, T ; D\left(S^{\alpha+1 / 2}\right)\right) \\
\left(u_{m}^{\prime \prime}\right) \quad \text { es acotada en } L^{\infty}\left(0, T ; S^{\alpha}\right)
\end{gathered}
$$

Luego, existe una subsucesión de $\left(u_{m}\right)$ que continuamos denotando de la misma forma tal que :

$$
\begin{aligned}
& u_{m} \rightarrow u \quad \text { debil - * en } \quad L^{\infty}\left(0, T ; D\left(S^{\alpha+1}\right)\right) \\
& u_{m}^{\prime} \rightarrow u^{\prime} \text { debil en } L^{2}\left(0, T ; D\left(S^{\alpha+1 / 2}\right)\right) \\
& u_{m}^{\prime \prime} \rightarrow u^{\prime \prime} \quad \text { debil - * en } L^{\infty}\left(0, T ; D\left(S^{\alpha}\right)\right)
\end{aligned}
$$

Por las inmersiones continuas y compactas: $D\left(A^{\alpha+1}\right) \subset D\left(A^{(2 \alpha+1) / 2}\right) \subset D\left(A^{\alpha}\right)$, y el Lema 2 , existe una adecuada subsucesión de $\left(u_{m}\right)$ que continuamos denotando de la misma forma, tal que :

$$
\begin{gathered}
u_{m} \stackrel{m \rightarrow \infty}{\longrightarrow} u \quad \text { fuerte } \quad \text { en } \quad \mathrm{C}^{0}\left([0, T] ; D\left(S^{(2 \alpha+1) / 2}\right)\right) \subset \mathrm{C}^{0}\left([0, T] ; W_{0}\right) \\
u_{m}^{\prime} \stackrel{m \rightarrow \infty}{\longrightarrow} u^{\prime} \quad \text { fuerte en } \quad \mathrm{C}^{0}\left([0, T] ; D\left(S^{\alpha}\right)\right) \subset \mathrm{C}^{0}\left([0, T] ; W_{1}\right)
\end{gathered}
$$

De (4.9), (4.10) y procediendo de forma estándar obtenemos que la función $u$ es solución del problema (3.1) y verifica

$$
u^{\prime \prime}+\psi(t) S u(t)=f(t) \quad \text { en } \quad L^{\infty}\left(0, T ; D\left(S^{\alpha}\right)\right)
$$

Para demostrar que la solución $u$ verifica el estimado de energía (3.12), probaremos primero que :

$$
u_{m}(t) \rightarrow u(t) \text { debil en } D\left(S^{\alpha+1}\right) \quad \forall t \in[0, T]
$$




$$
\begin{array}{cc}
u_{m}^{\prime}(t) \rightarrow u^{\prime}(t) \text { debil en } D\left(S^{(2 \alpha+1) / 2}\right) & \forall t \in[0, T] \\
u_{m}^{\prime \prime}(t) \rightarrow u^{\prime \prime}(t) \text { debil en } D\left(S^{\alpha}\right) & \forall t \in[0, T]
\end{array}
$$

Sea $v \in V_{k} ; m \geq k$. Tenemos

$$
\begin{gathered}
\left(S^{\alpha+1} u_{m}(t), S^{\alpha+1} v\right)=\left(S^{(2 \alpha+1) / 2} u_{m}(t), S^{(4 \alpha+3) / 4} v\right) \rightarrow \quad\left(S^{(2 \alpha+1) / 2} u(t), S^{(4 \alpha+3) / 4} v\right)= \\
=\left(S^{\alpha+1} u(t), S^{\alpha+1} v\right)
\end{gathered}
$$

Observación: Por (4.10)

$$
\begin{aligned}
& \left|\left(S^{(2 \alpha+1) / 2} u_{m}(t)-S^{(2 \alpha+1) / 2} u(t), S^{(4 \alpha+3) / 4} v\right)\right| \\
& \leq\left|S^{(2 \alpha+1) / 2} u_{m}(t)-S^{(2 \alpha+1) / 2} u(t)\right|\left|S^{(4 \alpha+3) / 4} v\right| \\
& \stackrel{m \rightarrow \infty}{\longrightarrow} 0
\end{aligned}
$$

Análogamente

$$
\begin{gathered}
\left(S^{(2 \alpha+1) / 2} u_{m}^{\prime}(t), S^{(2 \alpha+1) / 2} v\right)=\left(S^{(4 \alpha+1) / 4} u_{m}^{\prime}(t), S^{(4 \alpha+3) / 4} v\right) \rightarrow\left(S^{(4 \alpha+1) / 4} u^{\prime}(t), S^{(4 \alpha+3) / 4} v\right)= \\
=\left(S^{(2 \alpha+1) / 2} u^{\prime}(t), S^{(2 \alpha+1) / 2} v\right)
\end{gathered}
$$

Asimismo

$$
\begin{aligned}
& \left(S^{\alpha} u_{m}^{\prime \prime}(t), S^{\alpha} v_{k}\right)=-M(t)\left(S^{\alpha+1} u_{m}(t), S^{\alpha} v_{k}\right)+\left(S^{\alpha} f_{m}(t), S^{\alpha} v_{k}\right)= \\
& =-\psi(t)\left(S^{\alpha+1} u_{m}(t), S^{\alpha} v_{k}\right)+\left(f_{m}(t), S^{2 \alpha} v_{k}\right) \\
& =-\psi(t)\left(S^{\alpha+1} u_{m}(t), S^{\alpha} v_{k}\right)+\left(f(t), S^{2 \alpha} v_{k}\right) \rightarrow \\
& \quad-\psi(t)\left(S^{\alpha+1} u(t), S^{\alpha} v_{k}\right)+\left(S^{\alpha} f(t), S^{\alpha} v_{k}\right)=\left(S^{\alpha} u^{\prime \prime}, S^{\alpha} v_{k}\right)
\end{aligned}
$$

Luego por el Estimado 1, (4.16), (4.17) y (4.18), obtenemos que

$$
\begin{aligned}
& \quad\left|S^{\alpha} u^{\prime \prime}(t)\right|^{2}+\left|S^{(2 \alpha+1) / 2} u^{\prime}(t)\right|^{2}+\left|S^{\alpha+1} u(t)\right|^{2} \leq \\
& \quad \leq \frac{\lim _{m \rightarrow \infty}}{}\left\{\left|S^{\alpha} u_{m}^{\prime \prime}(t)\right|^{2}+\left|S^{(2 \alpha+1) / 2} u_{m}^{\prime}(t)\right|^{2}+\left|S^{\alpha+1} u_{m}(t)\right|^{2}\right\} \\
& \leq 2 M_{0}^{2} D_{0} e^{D_{1} t}+2 d_{2}^{2}|f|_{\infty}^{2}+D_{0} e^{D_{1} t}
\end{aligned}
$$

\section{Unicidad}

Sean $\mathrm{u}, \mathrm{z}$ dos soluciones de (3.1) ; $\mathrm{y}=\mathrm{z}$. Entonces

$$
\begin{gathered}
y \quad \in L^{\infty}\left(0, T ; D\left(S^{\alpha+1}\right)\right) \\
y^{\prime} \in L^{\infty}\left(0, T ; D\left(S^{(2 \alpha+1) / 2}\right)\right) \\
y^{\prime \prime} \in L^{2}\left(0, T ; D\left(S^{\alpha}\right)\right)
\end{gathered}
$$




$$
y(0)=0 ; \quad y^{\prime}(0)=0
$$

$y$ satisface la ecuación

$$
y^{\prime \prime}+\psi(t) A y=0 \quad \text { en } \quad L^{2}\left(0, T ; D\left(S^{\alpha}\right)\right)
$$

Componiendo con $w=2 y^{\prime}(t) \in D\left(S^{\alpha}\right)$ en esta ecuación, obtenemos

$$
\frac{d}{d t}\left\{\left|S^{\alpha} y^{\prime}(t)\right|^{2}+\psi(t)\left|S^{(2 \alpha+1) / 2} y(t)\right|^{2}\right\}=\psi^{\prime}(t)\left|S^{(2 \alpha+1) / 2} y(t)\right|^{2} \leq M_{1}\left|S^{(2 \alpha+1) / 2} y(t)\right|^{2}
$$

integrando de 0 a t y teniendo en cuenta que $y(0)=y^{\prime}(0)=0$ se obtiene

$$
\eta(t)=\left|S^{\alpha} y^{\prime}(t)\right|^{2}+m_{0}\left|S^{(2 \alpha+1) / 2} y(t)\right|^{2} \leq C \int_{0}^{t} \eta(s) d s
$$

y por el lema de Gronwall

$$
S^{\alpha} y^{\prime}(t)=S^{(2 \alpha+1) / 2} y(t)=0 \quad \forall t \in[0, T]
$$

Luego $u=z$.

\section{Problema no Lineal (Solución Local)}

En esta parte demostraremos la existencia de solución local del problema no-lineal.

$$
\begin{gathered}
u^{\prime \prime}+M\left(|u(t)|_{0},\left|u^{\prime}\right|_{1}^{2}\right) S u=f ; \text { en } L^{\infty}\left(0, T_{0} ; D\left(S^{\alpha}\right)\right) \\
u(0)=u_{0} ; u^{\prime}(0)=u_{1}
\end{gathered}
$$

donde $W_{0}, W_{1}$ son espacios de Banach, reales, reflexivos, tales que sus correspondientes espacios duales $W_{0}^{*}, W_{1}^{*}$ son estrictamente convexos.

$$
\begin{gathered}
D\left(S^{(2 \alpha+1) / 2}\right) \subset W_{0} . \\
D\left(S^{\alpha}\right) \subset W_{1} .
\end{gathered}
$$

Entonces de (6.3) y de la teoría de los operadores máximo monótonos, obtenemos las siguientes propiedades del operador de dualidad .

Teorema 2. Si $W$ es un espacio de Banach, real reflexivo tal que su espacio dual $W^{*}$, es estrictamente convexo. Entonces la aplicación de dualidad $J: W \rightarrow W^{*}$ es simple valuada, semicontinua, máximo monótona, acotada, coercitiva y

$$
|J u|_{W^{*}}=|u|_{W}
$$

La norma $u \rightarrow|u|_{W}$ es G-diferenciable sobre $W-\{0\}$ y si $\psi(u)=|u|_{W}$, entonces 


$$
\psi^{\prime}(u)=\frac{J u}{|u|_{W}} \quad \forall u \neq 0
$$

La función $u \rightarrow|u|_{W}^{2}$ es G-diferenciable sobre $W$ y si $\varphi(u)=|u|_{W}^{2}$, entonces

$$
\varphi^{\prime}(u)=2 J u \quad \forall u \in W
$$

\section{Hipótesis sobre la Función $M$}

H-1 $M \in C^{1}\left([0, T] \times[0, T] ; \mathbb{R}^{+}\right)$, donde $T$ es un número real positivo.

$\mathrm{H}-2 M(0,0)=0$.; y

H-3 Si $s>0$, entonces $M(s, r)>0, \forall r \geq 0$..

\section{Definición de Constantes}

Aqui definimos diversas constantes que aparecen en esta parte del trabajo.

Para datos iniciales $0 \neq u_{0}, u_{1}, f$ convenientes, consideramos:

$$
\begin{array}{ll}
C-1 & 0 \prec m_{0}=M\left(\frac{\left|u_{0}\right|_{W}}{2}, 0\right) \\
C-2 & C_{0}=\left|S^{(2 \alpha+1) / 2} u_{1}\right|^{2}+M\left(\left|u_{0}\right|_{W}\right)\left|S^{\alpha+1} u_{0}\right|^{2}+|f|_{L^{\infty}\left(0, T ; D\left(A^{(2 \alpha+1) / 2}\right)\right)}^{2} \\
C-3 & C_{1}=\min \left\{1, m_{0}\right\} \\
C-4 & D_{0}=\frac{C_{0}}{C_{1}}
\end{array}
$$

Consideremos las constantes de inmersión

$$
\begin{array}{lll}
C-5 & |v|_{0} \leq d_{0}\left|S^{\alpha+1} v\right| & \forall v \in D\left(S^{\alpha+1}\right) \\
C-6 & |v|_{0} \leq d_{1}\left|S^{(2 \alpha+1) / 2} v\right| & \forall v \in D\left(S^{(2 \alpha+1) / 2}\right) \\
C-7 & |v|_{1} \leq d_{2}\left|S^{\alpha} v\right| & \forall v \in D\left(S^{\alpha}\right) \\
C-8 & |v|_{1} \leq d_{3}\left|S^{(2 \alpha+1) / 2} v\right| & \forall v \in D\left(S^{(2 \alpha+1) / 2}\right) \\
C-9 & \left|S^{\alpha} v\right|_{1} \leq d_{4}\left|S^{(2 \alpha+1) / 2} v\right| & \forall v \in D\left(S^{(2 \alpha+1) / 2}\right)
\end{array}
$$

Desde la hipótesis (H.3) podemos considerar:

$$
\begin{array}{ll}
C-10 & M_{0}=\operatorname{Sup}\left\{M(s, r) /(s . r) \in\left[0, d_{0} K\right] \times\left[0, d_{1}^{2} K^{2}\right]\right\} \\
C-11 & M_{1}=\operatorname{Sup}\left\{M_{s}(s, r) /(s . r) \in\left[0, d_{0} K\right] \times\left[0, d_{1}^{2} K^{2}\right]\right\} \\
C-12 & M_{2}=\operatorname{Sup}\left\{M_{r}(s, r) /(s . r) \in\left[0, d_{0} K\right] \times\left[0, d_{1}^{2} K^{2}\right]\right\} \\
C-13 & C_{2}=\operatorname{Max}\left\{1, M_{0}\right\} \\
C-14 & D_{1}=\frac{C_{2}}{C_{1}} \\
C-15 & 0 \prec K^{2}=D_{0}\left(2 M_{0}^{2} e+d_{3}^{2}\right) \leq 1 \\
C-16 & 0 \prec T^{*}=\frac{\left|u_{0}\right|_{W}}{2 d_{2} K} \\
C-17 & T_{0}=\min \left\{T, T^{*}, \frac{1}{D_{1}}\right\}
\end{array}
$$


Consideremos el siguiente conjunto

$G=\left\{\begin{array}{l}v \in L^{\infty}\left(0, T_{0} ; D\left(S^{\alpha+1}\right)\right) / v^{\prime} \in L^{\infty}\left(0, T_{0} ; D\left(S^{2 \alpha+1 / 2}\right)\right), \quad v^{\prime \prime} \in L^{2}\left(0, T_{0} ; D\left(S^{\alpha}\right)\right) \\ v(0)=u_{0} \neq 0 \quad ;\left|S^{\alpha} v^{\prime \prime}(t)\right|^{2}+\left|S^{(2 \alpha+1) / 2} v^{\prime}(t)\right|^{2}+\left|S^{\alpha+1} v(t)\right|^{2} \leq K^{2} \quad \forall t \in\left[0 . T_{0}\right]\end{array}\right\}$

Lema 1 . Sí $v \in G$ y $\psi(t)=M\left(|v(t)|_{W}\right)$, entonces

$$
\begin{array}{cc}
0<m_{0} \leq \psi(t) & \forall t \in\left[0, T^{*}\right] \\
\left|\psi^{\prime}(t)\right| \leq M_{1} d_{1}+2 M_{2} d_{2} d_{3}=M_{4} \quad \forall t \in\left[0, T^{*}\right]
\end{array}
$$

Demostración. Sea $v \in G$, entonces

$$
\left.|| v(t)\right|_{0}-|v(0)|_{0}|\leq| v(t)-\left.v(0)\right|_{0}=\left|\int_{0}^{t} v^{\prime}(s) d s\right|_{0} \leq \int_{0}^{t}\left|v^{\prime}(s)\right|_{0} d s \leq d_{2} K t
$$

De esta desigualdad obtenemos :

$$
\left|u_{0}\right|_{0}-d_{2} K t=|v(0)|_{0}-d_{2} K t \leq|v(t)|_{0}
$$

Luego

$$
0 \prec \frac{\left|u_{0}\right|_{0}}{2}=\left|u_{0}\right|_{0}-\frac{\left|u_{0}\right|_{0}}{2} \leq\left|u_{0}\right|_{0}-d_{2} K T^{*} \leq\left|u_{0}\right|_{0}-d_{2} K t \leq|v(t)|_{0} \quad \forall t \in\left[0, T^{*}\right]
$$

Por la hipótesis H-2

$$
\psi(t)=M\left(|v(t)|_{0},\left|v^{\prime}(t)\right|_{1}^{2}\right) \geq M\left(\frac{\left|u_{0}\right|_{W}}{2}, 0\right)=m_{0} \succ 0
$$

lo que demuestra (8.1).

Debemos ahora obtener estimados para $\psi^{\prime}(t)$. Tenemos por la regla de la cadena, que

$$
\begin{gathered}
\psi^{\prime}(t)=M_{s}\left(|v(t)|_{0},\left|v^{\prime}(t)\right|_{1}^{2}\right)\left\langle\frac{J_{0} v(t)}{|v(t)|_{0}}, v^{\prime}(t)\right\rangle_{W_{0}^{*} \times W_{0}}+ \\
=2 M_{r}\left(|v(t)|_{0},\left|v^{\prime}(t)\right|_{1}^{2}\right)\left\langle J_{1} v^{\prime}(t), v^{\prime \prime}(t)\right\rangle_{W_{1}^{*} \times W_{1}}
\end{gathered}
$$

Entonces

$$
\begin{aligned}
& \left|\psi^{\prime}(t)\right| \leq M_{1}\left|v^{\prime}(t)\right|_{0}+2 M_{2}\left|v^{\prime}(t)\right|_{1}\left|v^{\prime \prime}(t)\right|_{1} \leq \\
& \leq M_{1} d_{1}\left|S^{(2 \alpha+1) / 2} v^{\prime}(t)\right|+2 M_{2} d_{2} d_{3}\left|S^{\alpha+1 / 2} v^{\prime}(t)\right|\left|S^{\alpha} v^{\prime \prime}(t)\right| \leq M_{1} d_{1}+2 M_{2} d_{2} d_{3}=M_{4}
\end{aligned}
$$

Teorema 2. Sean $v \in G$,

$$
0 \neq u_{0} \in D\left(S^{\alpha+1}\right)
$$




$$
\begin{gathered}
u_{1} \in D\left(S^{(2 \alpha+1) / 2}\right) \\
f \in L^{2}\left(0, T_{0} ; D\left(S^{(2 \alpha+1) / 2}\right)\right)
\end{gathered}
$$

Entonces, existe una única solución $u \in G$ del problema

$$
\begin{gathered}
u^{\prime \prime}+M\left(|v(t)|_{0},\left|v^{\prime}(t)\right|_{1}^{2}\right) S u=f \quad \text { en } L^{\infty}\left(0, T_{0} ; D\left(S^{\alpha}\right)\right) \\
u(0)=u_{0} ; u^{\prime}(0)=u_{1}
\end{gathered}
$$

Demostración : Procediendo como en la demostración del Teorema 1 , haciendo $T=T_{0}$ , $\psi(t)=M\left(|v(t)|_{0},\left|v^{\prime}(t)\right|_{1}^{2}\right)$ y utilizando el Lema 1 , se obtiene que para todo $v \in G$, existe una única función $u$, tal que:

$$
\begin{gathered}
u \quad \in L^{\infty}\left(0, T_{0} ; D\left(S^{\alpha+1}\right)\right) \\
u^{\prime} \in L^{\infty}\left(0, T_{0} ; D\left(S^{(2 \alpha+1) / 2}\right)\right) \\
u^{\prime \prime} \in L^{2}\left(0, T_{0} ; D\left(S^{\alpha}\right)\right) \\
u^{\prime \prime}+\psi(t) A u=f \quad \text { en } L^{\infty}\left(0, T ; D\left(S^{\alpha}\right)\right)
\end{gathered}
$$

y además satisface el siguiente estimado de energía

$$
\left|S^{\alpha} u^{\prime \prime}(t)\right|^{2}+\left|S^{(2 \alpha+1) / 2} u^{\prime}(t)\right|^{2}+\left|S^{\alpha+1} u(t)\right|^{2} \leq K^{2}
$$

Luego $u \in G$.

Teorema 3. Sean $u_{0}, u_{1}, f$, datos iniciales que satisfacen las condiciones del Teorema 2. Entonces existe una única solución $u \in G$ del problema (8.6)-(8.7).

Demostración. La idea es resolver una sucesión de problemas de la forma

$$
\begin{array}{cc}
z_{p}^{\prime \prime}+M\left(\left|z_{p-1}(t)\right|_{W}\right) A z_{p}=f \quad \text { en } & L^{2}\left(0, T_{0} ; D\left(S^{\alpha}\right)\right) \\
z_{p}(0)=u_{0} ; z_{p}^{\prime}(0)=u_{1} & p \geq 2
\end{array}
$$

donde $z_{2}$ es la única solución del problema

$$
\begin{gathered}
z_{2}^{\prime \prime}+M\left(\left|u_{0}\right|_{W}\right) A z_{2}=f \quad \text { en } L^{2}\left(0, T_{0} ; D\left(S^{\alpha}\right)\right) \\
z_{2}(0)=u_{0} ; z_{2}^{\prime}(0)=u_{1}
\end{gathered}
$$

Desde el Teorema 2, podemos definir una función $S: G \rightarrow G$ tal que $S z_{p-1}=z_{p}, p \geq 3$ donde $z_{p}$ es la única solución del problema (8.13)-(8.14).

Demostraremos la convergencia

$$
\begin{aligned}
& M\left(\left|z_{p-1}(t)\right|_{0},\left|z_{p-1}^{\prime}(t)\right|_{1}\right) S z_{p} \stackrel{p \rightarrow \infty}{\longrightarrow} M\left(|z(t)|_{0},\left|z^{\prime}(t)\right|_{1}\right) S z \\
& \quad \text { débil en } L^{\infty}\left(0, T_{0} ; D\left(S^{\alpha}\right)\right)
\end{aligned}
$$

En primer lugar probaremos que 


$$
M\left(\left|z_{p-1}\right|_{0},\left|z_{p-1}^{\prime}(t)\right|_{1}\right) \stackrel{p \rightarrow \infty}{\longrightarrow} M\left(|z|_{0},\left|z^{\prime}(t)\right|_{1}\right) \quad \text { en } \quad \mathrm{C}^{0}\left(\left[0, T_{0}\right]\right)
$$

En efecto;

desde que la sucesión $\left(z_{p}\right)_{p \geq 2} \subset G$, obtenemos que existe una función

$$
\begin{aligned}
& z_{p} \rightarrow z \quad \text { debil } * \text { en } L^{\infty}\left(0, T_{0} ; D\left(S^{\alpha+1}\right)\right) \\
& z_{p}^{\prime} \rightarrow z^{\prime} \quad \text { debil } * \text { en } L^{\infty}\left(0, T_{0} ; D\left(S^{(2 \alpha+1) / 2}\right)\right) \\
& z_{p}^{\prime \prime} \rightarrow z^{\prime \prime} \quad \text { debil en } L^{2}\left(0, T_{0} ; D\left(S^{\alpha}\right)\right)
\end{aligned}
$$

además por el Lema 2

$$
\begin{gathered}
z_{p-1} \stackrel{p \rightarrow \infty}{\longrightarrow} z \quad \text { en } \quad \mathrm{C}\left(\left[0, T_{0}\right] ; D\left(S^{(2 \alpha+1) / 2}\right)\right) \subset \mathrm{C}\left(\left[0, T_{0}\right] ; W_{0}\right) \\
z_{p-1}^{\prime} \stackrel{p \rightarrow \infty}{\longrightarrow} z^{\prime} \\
\text { en } \quad \mathrm{C}\left(\left[0, T_{0}\right] ; D\left(S^{\alpha}\right)\right) \subset C\left(\left[0, T_{0}\right] ; W_{1}\right)
\end{gathered}
$$

Luego, haciendo

$$
\begin{aligned}
N_{p}(t)=M\left(\left|z_{p-1}(t)\right|_{0},\left|z_{p-1}(t)\right|_{1}^{2}\right) & ; N(t)=M\left(|z(t)|_{0},|z(t)|_{1}^{2}\right) \\
\left|\int_{0}^{T}\left(N_{p}(t) S z_{p}(t)-N(t) S z(t), v(t)\right)_{\alpha}\right| d t \leq & \left|\int_{0}^{T}\left(N_{p}(t) S z_{p}(t)-N(t) S z_{p}(t), v(t)\right)_{\alpha}\right|_{d} d t \\
& +\left|\int_{0}^{T}\left(N(t) S_{p} z(t)-N(t) S z(t), v(t)\right)_{\alpha}\right| d t
\end{aligned}
$$

desde que la función $N \in C\left(\left[0, T_{0}\right]\right)$ y es acotada , $N v \in L^{1}\left(0, T_{0}, D\left(S^{\alpha}\right)\right)$. Luego por $\left|\int_{0}^{T}\left(N_{p}(t) S z_{p}(t)-N(t) S z_{p}(t), v(t)\right)_{\alpha}\right| d t \leq \int_{0}^{T}\left|N_{p}(t)-N(t)\right|\left|S^{\alpha+1} z_{p}(t)\right|\left|S^{\alpha} v(t)\right| d t \leq$ $\leq \sup _{t \in\left[0, T_{0}\right]}\left|N_{p}(t)-N(t)\right|\left|S^{\alpha+1} z_{p}\right| \int_{0}^{T}\left|S^{\alpha}(t)\right| d t \leq C \sup _{t \in\left[0, T_{0}\right]}\left|N_{p}(t)-N(t)\right| \stackrel{p \rightarrow \infty}{\longrightarrow} 0$ lo que demuestra (8.18). Luego

$$
z^{\prime \prime}+M\left(|z(t)|_{0},\left|z^{\prime}(t)\right|_{1}^{2}\right) S z=f \quad \text { en } L^{\infty}\left(0, T ; D\left(S^{\alpha}\right)\right)
$$

Procediendo como en la demostración del Teorema 1, se prueba que :

$$
\begin{array}{ccc}
z_{p}(t) \rightarrow z(t) & \text { debil en } D\left(S^{\alpha+1}\right) & \forall t \in\left[0, T_{0}\right] \\
z_{p}^{\prime}(t) \rightarrow z^{\prime}(t) \quad \text { debil en } D\left(S^{(2 \alpha+1) / 2}\right) & \forall t \in\left[0, T_{0}\right] \\
z_{p}^{\prime \prime}(t) \rightarrow z^{\prime \prime}(t) \quad \text { debil en } D\left(S^{\alpha}\right) & \forall t \in\left[0, T_{0}\right]
\end{array}
$$

Entonces

$$
\begin{aligned}
\left|S^{\alpha} z^{\prime \prime}(t)\right|^{2}+ & \left|S^{\alpha+1 / 2} z^{\prime}(t)\right|^{2}+\left|S^{\alpha+1} z(t)\right|^{2} \leq \\
& \leq \frac{\lim _{p \rightarrow \infty}}{2}\left\{\left|S^{\alpha} z_{p}(t)\right|^{2}+\left|S^{(2 \alpha+1) / 2} z_{p}^{\prime}(t)\right|^{2}+\left|S^{\alpha+1} z_{p}(t)\right|^{2}\right\} \leq K^{2}
\end{aligned}
$$

por lo tanto $z \in G$. 


\section{Unicidad}

Sean $u, z$ dos soluciones del problema (8.6), (8.7) y $y=u-z$. Entonces se verifica que y $\in L^{\infty}\left(0, T_{0} ; D\left(S^{\alpha+1}\right)\right)$

$\mathrm{y}^{\prime} \in \quad L^{\infty}\left(0, T_{0} ; D\left(S^{2 \alpha+1 / 2}\right)\right)$

$\mathrm{y}^{\prime \prime} \in \quad L^{\infty}\left(0, T_{0} ; D\left(S^{\alpha}\right)\right)$

$\mathrm{y}(0)=y^{\prime}(0)=0$

y satisface la ecuación

$$
y^{\prime \prime}+M\left(|u|_{0},\left|u^{\prime}\right|_{1}^{2}\right) S y=\left(M\left(|u|_{0},\left|u^{\prime}\right|_{1}^{2}\right)-M\left(|z|_{0},\left|z^{\prime}\right|_{1}^{2}\right)\right) S z \quad \text { en } \quad L^{2}\left(0, T_{0} ; D\left(S^{\alpha}\right)\right)
$$

Componiendo con $w(t)=2 y^{\prime}(t)$ en esta ecuación y teniendo en cuenta que

$$
\left|M\left(|z(t)|_{0},\left|z^{\prime}(t)\right|_{1}\right)-M\left(|u(t)|_{0},\left|u^{\prime}(t)\right|_{1}\right)\right| \leq C|y(t)|_{0}+C_{1}\left|y^{\prime}(t)\right|_{1}
$$

obtenemos

$$
\begin{aligned}
\frac{d}{d t}\left\{\left|S^{\alpha} y^{\prime}(t)\right|^{2}+\psi(t)\left|S^{(2 \alpha+1) / 2} y(t)\right|^{2}\right\} \leq & C_{0}|y(t)|_{0}\left|S^{\alpha} z(t)\right|\left|S^{\alpha} y^{\prime}(t)\right|+ \\
& +C_{1}\left|y^{\prime}(t)\right|_{1}\left|S^{\alpha} z(t)\right|\left|S^{\alpha} y^{\prime}(t)\right| \\
& +\psi^{\prime}(t)\left|S^{(2 \alpha+1) / 2} y(t)\right|^{2} \\
\leq & C_{2}\left|S^{(2 \alpha+1) / 2} y(t)\right|\left|S^{\alpha} y^{\prime}(t)\right|+C_{3}\left|S^{\alpha} y^{\prime}(t)\right|^{2} \\
& +C_{4}\left|S^{(2 \alpha+1) / 2} y(t)\right|^{2} \\
\leq & C\left|S^{\alpha} y^{\prime}(t)\right|^{2}+C\left|S^{(2 \alpha+1) / 2} y(t)\right|^{2}
\end{aligned}
$$

donde $C$ representa diversas constantes que no dependen de $t$. Integrando de 0 a t y teniendo en cuenta que $y(0)=y^{\prime}(0)=0$ obtenemos

$$
\eta(t)=\left|S^{\alpha} y^{\prime}(t)\right|^{2}+m_{0}\left|S^{(2 \alpha+1) / 2} y(t)\right|^{2} \leq C \int_{0}^{t} \eta(s) d s
$$

y por el lema de Gronwall $S^{\alpha} y^{\prime}(t)=S^{(2 \alpha+1) / 2} y(t)=0 \quad \forall t \in\left[0, T_{0}\right]$. Luego $u=z$.

\section{Aplicaciones}

1. Sea $\Omega \subset \mathbb{R}^{n}$ un abierto regular . Consideremos $: \rho: \Omega \rightarrow \mathbb{R}^{+}$, continua, positiva en casi todo punto y acotada superiormente digamos $0<\rho(x) \leq \rho_{1}$

$$
\begin{gathered}
V=H_{0}^{1}(\Omega), W=L^{p}(\Omega), H=L^{2}(\Omega) \\
1<p \leq \frac{2 n}{n-2}, \text { si } n \geq 3, \text { y } 1<p<\infty, \text { si } n=1,2
\end{gathered}
$$




$$
\begin{gathered}
M(s, r)=a+b s^{q}+c r, q \geq 1, a \geq 0, b \succ 0 ; c \geq 0 . \\
A u=-\Delta u, B u=\rho u,
\end{gathered}
$$

Entonces existe una única solución del problema

$$
\left\{\begin{array}{lr}
\rho(x) \frac{\partial^{2} u}{\partial t^{2}}+\left(a+b \int_{\Omega}|\nabla u(x, t)|^{p} d x+c \int\left|u^{\prime}(x, t)\right|^{2} d x\right)(-\Delta u)=f & \text { en } Q \\
u=0 & \text { en } \Gamma \\
u(0)=u_{0} \quad ; \quad u^{\prime}(0)=u_{1} & \text { en } \Omega
\end{array}\right.
$$

2. Sea $M(s)=N\left(s^{2}\right)$ y $J: W \rightarrow W^{*}$ el operador de dualidad sobre $W$. Consideramos el problema

$$
\left\{\begin{array}{l}
u^{\prime \prime}+N\left(|u|_{W}^{2}\right) A u^{\cdot}=f \\
u(0)=u_{0} ; u^{\prime}(0)=u_{1}
\end{array}\right.
$$

En este caso si $\varphi(u)=|u|_{W}^{2}$, entonces $\varphi^{\prime}(u)=2 J(u), \forall u \in W$, lo que facilita los cálculos desde que no requiere que $u \neq 0$.

En el caso que $W$ sea un espacio de Hilbert $\varphi^{\prime}(u)=2 J u$, donde $J$ es el operador de Riesz , $\langle J u, v\rangle_{W^{*} \times W}=(u, v)_{W} ; \forall u, v \in W$.

Como casos particulares de la aplicación 2, para $W=V=D\left(A^{1 / 2}\right)$, tenemos el problema abstracto de Kirchhoff

$$
\left\{\begin{array}{l}
u^{\prime \prime}+N\left(\left|A^{1 / 2} u\right|^{2}\right) A u=f \\
u(0)=u_{0} ; u^{\prime}(0)=u_{1}
\end{array}\right.
$$

formulado por Lions J.L. en [9]. Si $W=H$, se tiene el problema de Carrier abstracto

$$
\left\{\begin{array}{l}
u^{\prime \prime}+N\left(|u|_{H}^{2}\right) A u=f \\
u(0)=u_{0} ; u^{\prime}(0)=u_{1}
\end{array}\right.
$$

Una adecuada generalización de las aplicaciones (1) y (2) es considerar $W=D\left(A^{\alpha}\right)$ para obtener

$$
\left\{\begin{array}{l}
u^{\prime \prime}+N\left(\left|A^{\alpha} u\right|^{2}\right) A u=f \\
u(0)=u_{0} ; u^{\prime}(0)=u_{1}
\end{array}\right.
$$

conocido como el modelo abstracto de Kirchhoff-Carrier. 


\section{Referencias}

[1] AROSIO A -SPAGNOLO S..- "Global solutions of the Cauchy problem for a non-linear Hyperbolic Equation ".Universita di Pisa . Departamento de Matematica. Roma (1982).

[2] CARRIER G.F. "On the non-linear vibration problem of the elastic string" Quart. Appl. Math. - 3 - pp:157-165 (1945).

[3] COUSIN A. ,FROTA C., LARKIN N. ,MEDEIROS L.A. "On the abstrac model of Kirchhoff-Carrier Equation". Comm. In App. Analysis. - 3 - (1997).

[4] CRIPPA H. "On Local Solutions of Some mildly Degenerate Hyperbolic Equations" Non-linear Analysis, vol. 21 (8)(1993).

[5] EBIHARA Y.-MEDEIROS L.A. -.MILLA.M- "Local solutions for a nonlinear degenerate hyperbolic equations". Nonlinear Analysis.Vol.10. pp 27-40 (1986).

[6] IZAGUIRRE R. FUENTES R. MILLA M . "Existente of local solutions of the Kirchhoff - Carrier equation in Banach spaces" Theory, Methods and Applications (2007).

[7] IZAGUIRRE R. - GARCIA - "Solución Local para una clase de ecuaciones no lineales de tipo Kirchhoff-Carrier" 44 Seminario Brasilero de Anàlisis (1998)

[8] IZAGUIRRE R. - VELIZ V. "Solucion local para una clase de ecuaciones no-lineales degeneradas tipo Kirchhoff- Carrier" . I Seminario Internacional de Ecuaciones Diferenciales y Aplicaciones. Universidad Ricardo Palma - Lima -Perú- (1999).

[9] IZAGUIRRE R.-VELIZ V. "Solución Local para una clase de ecuaciones no-lineales de tipo Kirchoff". Actas del 45 Seminario Brasileiro de Analise (1997).

[10] KIRCHHOFF G. "Vorlesungenuber mechanik"ch. 9 §7 Taubner, Leipzig (1883).

[11] LIONS J.L. "Quelques Methodes de Resolution des Probleme aux limites nonlinear".Dunod.Paris, (1969).

[12] LONG N. "Non linear Kirchhoff-Carrier Wave Equation in a unit membrane with mixed homogenous boundary conditions" Electronic Journal of Differential Equations . Vol. 138, pp 1-18 (2005).

[13] MEDEIROS L.A. - MILLA M. "Solutions for the Equation of Nonlinear Vibrations Sobolev Spaces of Fractionary Order" Math. Apl. Comp. 6 (1987).

[14] MEDEIROS L.A. -.MILLA M. "Remarks on a nonlinear model vibrations of string with damping".30 Seminario Brasileiro de Analise.L.N.C.C.-R.J. (1989).

[15] ONO K. " Global existence,Decay,and Blowup of Solutions for Some Mildly Degenerate Nomlinear Kirchhoff String” J. Diff. Eq. 137 (1997). 
[16] PERLA G.- "On classical solutionsof a quasilinear hyperbolic equations". Nonlinear Analysis.Vol.3.PP 613-627 (1979).

[17] POHOZAEV S.- "The Kirchoff quasilinear hyperbolic equation ". Differential Equations Vol.21 pp. 101-107 (1985).

[18] POHOZAEV S. "On a class of quasilinear hyperbolic equations" Math. Sbornik, Vol 96 PP. 152-156 (1975).

[19] RIVERA P. "On local strong solutions of a nonlinear partial differential equation".Appl. Analysis vol.10. (1980).

[20] SIMON J. Compact Sets in the Space $L^{p}(0, T ; B)$. Universite Pierre et Marie Curie. Laboratoire dÁnalyse Numerique (1985).

[21] YAMADA Y. "Some Nonlinear Degenerate Wave Equations" Non Linear Analysis, 10 (11) (1987).

[22] ZEIDLER E. "Non Linear Functional Analysis" Part II-B (1990). 